\title{
Rationale and Trial Design of Bardoxolone Methyl Evaluation in Patients with Chronic Kidney Disease and Type 2 Diabetes: The Occurrence of Renal Events (BEACON)
}

\author{
Dick de Zeeuw ${ }^{a}$ Tadao Akizawa ${ }^{b}$ Rajiv Agarwalc Paul Audhyad \\ George L. Bakris $^{\mathrm{e}}$ Melanie Chin ${ }^{d}$ Melissa Krauth ${ }^{d}$ Hiddo J. Lambers Heerspink ${ }^{a}$ \\ Colin J. Meyer ${ }^{d}$ John J. McMurray ${ }^{f}$ Hans-Henrik Parving ${ }^{g}$ Pablo E. Pergolah \\ Giuseppe Remuzzi ${ }^{i} \quad$ Robert D. Toto ${ }^{j} \quad$ Nosratola D. Vazirik ${ }^{k}$ Christoph Wanner \\ David G. Warnock ${ }^{m}$ Janet Wittes ${ }^{n}$ Glenn M. Chertow ${ }^{\circ}$

\begin{abstract}
a Department of Clinical Pharmacology, University of Groningen, University Medical Center, Groningen,

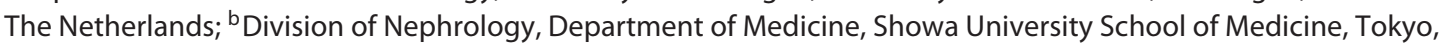
Japan; ${ }^{C}$ Department of Medicine, Indiana University School of Medicine and Richard L. Roudebush Veterans Affairs Medical Center, Indianapolis, Ind., ' REATA Pharmaceuticals, Irving, Tex., and ' Department of Medicine, AHS Comprehensive Hypertension Center, The University of Chicago Medicine, Chicago, III., USA;

${ }^{f}$ BHF Cardiovascular Research Centre, University of Glasgow, Glasgow, UK; ${ }^{9}$ Department of Medical Endocrinology, Rigshospitalet, University of Copenhagen, Copenhagen, Denmark; ${ }^{\mathrm{h}}$ Renal Associates, PA, San Antonio, Tex., USA; 'Unit of Nephrology, Azienda Ospedaliera Ospedali Riuniti di Bergamo and Mario Negri Institute for Pharmacological Research, Bergamo, Italy; ${ }^{j}$ Department of Medicine, University of Texas Southwestern Medical Irvine, Calif., USA; 'Division of Nephrology, Department of Medicine, University of Würzburg, Würzburg, Germany; mDepartment of Medicine, University of Alabama at Birmingham, Birmingham, Ala., ${ }^{\text {n}}$ Statistics Collaborative, Inc., Washington, D.C., and ${ }^{\circ}$ Department of Medicine, Stanford University School of Medicine, Palo Alto, Calif., USA
\end{abstract} \\ Center, Dallas, Tex., and kDivision of Nephrology and Hypertension, Department of Medicine, University of California,
}

\section{Key Words}

Clinical trial · Trial design · Bardoxolone methyl · Nrf2 · End-stage renal disease - Cardiovascular death - Chronic kidney disease $\cdot$ Diabetes mellitus · Glomerular filtration rate

\footnotetext{
Abstract

Background: Chronic kidney disease (CKD) associated with type 2 diabetes mellitus constitutes a global epidemic complicated by considerable renal and cardiovascular morbidity
}

and mortality, despite the provision of inhibitors of the renin-angiotensin-aldosterone system (RAAS). Bardoxolone methyl, a synthetic triterpenoid that reduces oxidative stress and inflammation through Nrf2 activation and inhibition of NF-KB was previously shown to increase estimated glomerular filtration rate (eGFR) in patients with CKD associated with type 2 diabetes mellitus. To date, no antioxidant or anti-inflammatory therapy has proved successful at slowing the progression of CKD. Methods: Herein, we describe the design of Bardoxolone Methyl Evaluation in Patients with Chronic Kidney Disease and Type 2 Diabetes: the Occurrence

\section{KARGER}

E-Mail karger@karger.com

www.karger.com/ajn
(C) 2013 S. Karger AG Basel

$0250-8095 / 13 / 0373-0212 \$ 38.00 / 0$
Dick de Zeeuw, MD, PhD

Department of Clinical Pharmacology

University Medical Center Groningen

Ant Deusinglaan 1, NL-9713 AV Groningen (The Netherlands)

E-Mail d.de.zeeuw@umcg.nl 
of Renal Events (BEACON) trial, a multinational, multicenter, double-blind, randomized, placebo-controlled Phase 3 trial designed to determine whether long-term administration of bardoxolone methyl (on a background of standard therapy, including RAAS inhibitors) safely reduces renal and cardiac morbidity and mortality. Results: The primary composite endpoint is time-to-first occurrence of either end-stage renal disease or cardiovascular death. Secondary endpoints include the change in eGFR and time to occurrence of cardiovascular events. Conclusion: BEACON will be the first eventdriven trial to evaluate the effect of an oral antioxidant and anti-inflammatory drug in advanced CKD.

Copyright $\odot 2013$ S. Karger AG, Basel

\section{Introduction}

Type 2 diabetes mellitus prevalence is increasing worldwide. Sequelae vary among affected persons but, in general, multiple treatment strategies are required to prevent or halt progressive multiorgan disease. Diabetes is the most common cause of end-stage renal disease (ESRD), which occurs in $~ 30 \%$ of all patients globally [1]. Loss of kidney function is progressive and associated with high cardiovascular (CV) and renal morbidity and mortality, equaling or exceeding mortality of many treated cancers [2].

Several treatments, such as drugs that block the reninangiotensin-aldosterone system (RAAS), can reduce or postpone renal and $\mathrm{CV}$ events in patients with chronic kidney disease (CKD) and type 2 diabetes [3-7]. However, despite relative risk reductions of $15-30 \%$ for composite renal outcomes (i.e. doubling of serum creatinine, dialysis or renal transplantation, and death) and $20-30 \%$ for slowing progression to ESRD, residual risk remains extremely high and mean time to dialysis is delayed by $<6$ months. Additional therapeutic strategies or interventions to attenuate, arrest, or reverse disease progression are needed.

In recent years, several approaches, including endothelin receptor antagonists (e.g. avosentan), glucosaminoglycans (e.g. sulodexide), advanced glycation endproduct (AGE) inhibitors (e.g. pyridorin), erythropoiesis-stimulating agents (e.g. darbepoetin alpha), HMGCoA reductase inhibitors alone or in combination with other cholesterol-lowering agents (e.g. simvastatin + ezetimibe) and RAAS inhibitor combination strategies (e.g. angiotensin-converting enzyme inhibition (ACEi), plus angiotensin receptor blockade (ARB) or ACEi or $A R B$ plus a direct renin inhibitor (DRI)) have produced generally disappointing results [8-14].
More recently, progressive kidney function loss in diabetes mellitus has been recognized, at least in part, to be inflammatory in origin [15-19]. The association between inflammation and progressive kidney disease has directed attention toward new pathways of intervention. Compelling experimental and early-phase clinical data showed bardoxolone methyl, a synthetic triterpenoid that reduces oxidative stress through Nrf2 activation and supports resolution of inflammation through NF- $\kappa B$ inhibition, increases estimated glomerular filtration rate (eGFR) in patients with type 2 diabetes mellitus and stage $3 \mathrm{~b} / 4 \mathrm{CKD}$ $[20,21]$. These findings raise the possibility that bardoxolone methyl could delay the need for dialysis or transplantation and/or reduce CV complications in patients with type 2 diabetes mellitus and progressive CKD. However, the eGFR increase could also reflect an increase in intraglomerular pressure and thus a potential risk of hyperfiltration-related kidney injury.

Validating the effect of bardoxolone methyl on eGFR requires study of the drug's effects on clinically meaningful endpoints. Primary outcomes of most CKD progression trials typically include the classic composite endpoint consisting of time-to-first occurrence of doubling of serum creatinine, ESRD, or death. However, since bardoxolone methyl reduces serum creatinine levels, a renal endpoint that includes serum creatinine change could be considered inappropriate, leaving initiation of dialysis or death as the most clinically relevant endpoints. For feasibility and practicality the study population of such a trial should include patients with advanced (stage 4) CKD (eGFR 15 to $<30 \mathrm{ml} / \mathrm{min} / 1.73 \mathrm{~m}^{2}$ ). No other trial has tested an agent with the aim of delaying progression to ESRD in patients with such advanced CKD.

This communication describes the design of the Bardoxolone Methyl Evaluation in Patients with Chronic Kidney Disease and Type 2 Diabetes Mellitus: the Occurrence of Renal Events (BEACON) trial, which will test the effects of bardoxolone methyl against placebo on the risk of ESRD and CV death in patients with type 2 diabetes mellitus and stage $4 \mathrm{CKD}$ receiving standard of care. We describe specific challenges faced in designing such a tri$\mathrm{al}$, in which the intervention appears to alter an intermediate functional measurement (in this case, serum creatinine or eGFR) that may influence the 'hard' outcome (dialysis or transplantation), and how we incorporated data from previously conducted clinical trials in earlier CKD stages to inform our sample size and estimated event rate. 
Table 1. Inclusion and exclusion criteria of the study population

Inclusion criteria

Screening eGFR $\geq 15.0$ and $<30.0 \mathrm{ml} / \mathrm{min} / 1.73 \mathrm{~m}^{2}$

History of type 2 diabetes mellitus

$\geq 18$ years of age

Stable treatment with an ACE inhibitor and/or ARB for at least 6 weeks prior to and during screening, unless medically contraindicated with documentation

$\mathrm{SBP} \leq 160 \mathrm{~mm} \mathrm{Hg}$ and $\geq 105 \mathrm{~mm} \mathrm{Hg}$ DBP $\leq 90 \mathrm{~mm} \mathrm{Hg}$

Use of birth control methods during screening, on study, and for at least 30 days after the last dose of study drug

Serum magnesium $\geq 1.3 \mathrm{mEq} / \mathrm{l}(0.65 \mathrm{mmol} / \mathrm{l})$ at screening

Exclusion criteria

Type 1 diabetes mellitus

Known non-diabetic renal disease

Ongoing clinical investigation with evidence suggesting non-diabetic renal disease other than nephrosclerosis

History of a renal transplant or a planned transplant from a living donor

Albumin/creatinine ratio (UACR) $>3,500 \mathrm{mg} / \mathrm{g}(395.5 \mathrm{mg} / \mathrm{mmol})$ at screening

Hemoglobin $\mathrm{A}_{1 \mathrm{c}}$ level $>11.0 \%$ (97 $\left.\mathrm{mmol} / \mathrm{mol}\right)$ at screening

Acute dialysis or acute kidney injury within 12 weeks prior to or during screening

Clinical signs and/or symptoms of uremia and expected need for renal replacement therapy within 12 weeks following randomization, as assessed by the investigator

Recently active cardiovascular disease defined as:

Unstable angina pectoris within 12 weeks before study randomization

Myocardial infarction, coronary artery bypass graft surgery, or percutaneous transluminal coronary angioplasty/stent within 12 weeks before study randomization

Cerebrovascular accident, including transient ischemic attack within 12 weeks before study randomization

Current diagnosis of Class III or IV NYHA congestive heart failure

Clinical diagnosis of severe obstructive valvular heart disease or severe obstructive hypertrophic cardiomyopathy

Atrioventricular block, $2^{\circ}$ or $3^{\circ}$, not successfully treated with a pacemaker

Administration of a contrast agent that may induce nephropathy within 30 days prior to study randomization or planned during the study

Systemic immunosuppression for more than 2 weeks, cumulatively, within the 12 weeks prior to randomization or anticipated need for immunosuppression during the study

Total bilirubin, aspartate transaminase (AST), or alanine transaminase (ALT) level greater than the upper limit of normal (ULN) or alkaline phosphatase level greater than two times the ULN on ANY screening laboratory test result
Female patients who are pregnant, intend to become pregnant during the study, or are nursing

BMI $<18.5$

Known hypersensitivity to any component of the study drug

Current history of drug or alcohol abuse, as assessed by the investigator

Clinically significant infection requiring intravenous administration of antibiotics or hospitalization within 6 weeks prior to screening

Diagnosis or treatment of a malignancy in the past 5 years, excluding non-melanoma skin cancer and carcinoma in situ of the cervix or a condition highly likely to transform into a malignancy during the course of the study

A clinical condition that, in the judgment of the investigator, could potentially pose a health risk to the subject while involved in the study

Participation in a clinical study involving any intervention within 30 days prior to randomization, concurrent participation in such a study, or participation in a prior clinical study involving bardoxolone methyl in any form

\section{BEACON Design}

\section{Study Population}

The study population includes approximately 2,000 patients with type 2 diabetes mellitus defined as onset at $\geq 30$ years of age; if diagnosed at a younger age, fasting Cpeptide level was used to confirm type 2 diabetes mellitus. Patients should have stage $4 \mathrm{CKD}$, defined as eGFR of 15 to $<30 \mathrm{ml} / \mathrm{min} / 1.73 \mathrm{~m}^{2}$. See table 1 for additional inclusion and exclusion criteria.

\section{Study Outcomes}

The primary efficacy aim of BEACON is to assess efficacy of bardoxolone methyl relative to placebo in delaying progression to ESRD (maintenance dialysis, kidney transplantation or kidney death (i.e. loss of kidney function with refusal of dialysis or transplantation)) or $\mathrm{CV}$ death. The composite primary outcome includes $\mathrm{CV}$ death because it is an important competing risk in these patients. Non-CV and non-renal mortality were not included in the primary endpoint as they are not assumed to be related to kidney disease progression.

Secondary efficacy endpoints of BEACON are (1) change in eGFR over the study duration, (2) time-to-first hospitalization for heart failure or death due to heart failure, and (3) time-to-first event in a composite CV endpoint consisting of $\mathrm{CV}$ death, non-fatal myocardial infarction, non-fatal stroke, and hospitalization for heart failure. 
Fig. 1. BEACON design overview and study objectives. $\mathrm{R}=$ Randomization; SDT = study drug termination; $\mathrm{SDD}=$ spray-dried dispersion. * During week 4, patients will begin in-person visits every 4 weeks until week 20. Beginning week 20, telephone contact will alternate with inperson site visits every 4 weeks for the duration of the study. Patients will undergo more extensive evaluations during semiannual visits at week 24 and every 24 weeks thereafter.

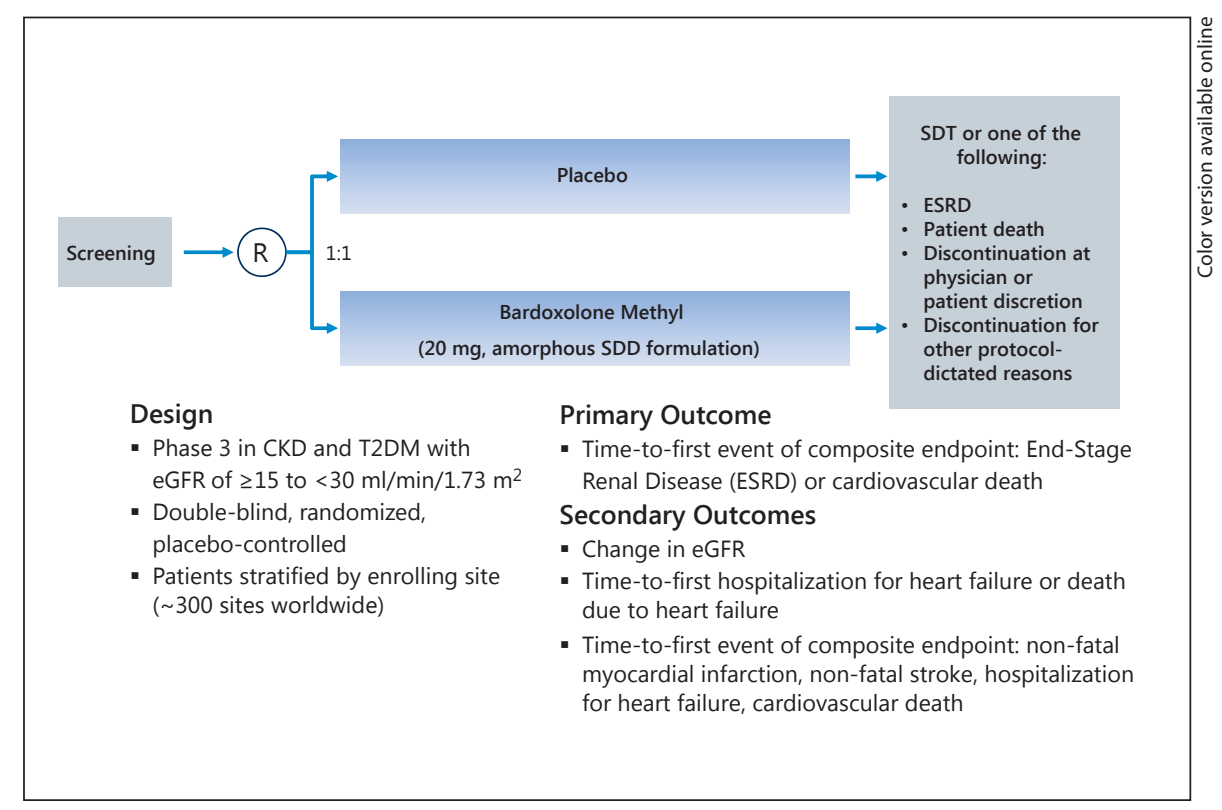

\section{Study Design}

BEACON is a multinational, multicenter, randomized, double-blind, placebo-controlled, parallel-group Phase 3 study comparing the efficacy and safety of bardoxolone methyl relative to placebo in patients with type 2 diabetes mellitus and stage 4 CKD receiving standard of care. Study patients who met all inclusion criteria and no exclusion criterion and satisfied screening evaluations were randomized 1:1 to receive placebo or $20 \mathrm{mg}$ bardoxolone methyl (amorphous spray-dried dispersion (SDD) formulation; fig. 1). Patients are to take one study drug capsule orally each morning on an empty stomach ( $1 \mathrm{~h}$ before or $2 \mathrm{~h}$ after eating). Randomization was stratified by enrollment site. To ensure a balanced study population at risk for ESRD, a maximum of $25 \%$ of all enrolled patients may demonstrate a urine albumin-to-creatinine ratio (UACR) $<30 \mu \mathrm{g} / \mathrm{mg}$ ( $3.39 \mathrm{mg} / \mathrm{mmol})$. To ensure the study population reflects patients currently following clinical practice guidelines treatment, a maximum of 1 in 8 (12.5\%) of patients are permitted to be off ACEi or ARB treatment (or not on a K/DOQI goal dose of ACEi or ARB) at the start of study for a medical contraindication (e.g. hyperkalemia, hypotension, angioedema).

\section{Follow-Up, Study Visits and Management}

Patients are assessed every 4 weeks throughout the trial. After randomization, patients are seen every 4 weeks through to Week 16 (fig. 1), after which in-person visits alternate with telephone contact visits every 4 weeks. At
Week 24 and every 24 weeks thereafter, each subject undergoes a more extensive evaluation (fig. 1).

Blood pressure is measured 3 times at each visit with an oscillometric automated device (SunTech 247) provided by the sponsor. Consistent with current clinical practice guidelines for management of hypertension in patients with type 2 diabetes mellitus, investigators are instructed to target blood pressure to a level of $\leq 130 / 80 \mathrm{~mm} \mathrm{Hg}$ for patients with an UACR $\geq 300 \mathrm{mg} / \mathrm{g}(33.9 \mathrm{mg} / \mathrm{mmol})$ and $\leq 140 / 90 \mathrm{~mm} \mathrm{Hg}$ for patients with an UACR $\leq 300 \mathrm{mg} / \mathrm{g}$ $(33.9 \mathrm{mg} / \mathrm{mmol})$. The protocol discourages change or discontinuation of either ACEi or ARB treatment, unless required secondary to development of a medical contraindication. For management of all other comorbidities, investigators are instructed to follow standard of care.

At each in-person visit, symptoms considered related to uremia are systematically captured, and for each subject requiring maintenance dialysis, primary and secondary reasons for initiating dialysis are obtained (table 2). Patients are to receive study drug until they reach ESRD, they die, they or the investigator decides to discontinue study drug, they withdraw consent to participate in the study, or they meet additional protocol-dictated reasons that warrant discontinuing study drug. Otherwise, patients are to continue receiving study drug until the study drug termination (SDT) date, as defined below. Even after study drug discontinuation, patients are to return for in-person and/or participate in telephone visits to capture potential primary or secondary endpoints. 
Table 2. Indications for initiation of dialysis (indications for dialysis initiation will be captured at each visit on a standardized case report form, marked as primary or secondary, and classified according to the listed classification terms)

\section{Primary or secondary indications for initiation of dialysis Nausea/vomiting \\ Fatigue \\ Deterioration in nutritional status/recent significant weight loss \\ Neurologic dysfunction (neuropathy, encephalopathy, psychiatric disturbances, seizures) \\ Volume overload \\ Hyperkalemia \\ Metabolic acidosis \\ Hyperphosphatemia \\ Hypercalcemia/hypocalcemia \\ Anemia \\ Azotemia \\ Bleeding diathesis \\ Pleuritis/pericarditis of uremic origin \\ Malignant hypertension/uncontrolled hypertension \\ Intractable sleep disturbances \\ Intractable anhedonia}

Classification

New, urgent

Refractory to treatment

Worsening

Severe

The study protocol was designed and implemented in accordance with the ICH Harmonized Tripartite Guidelines for Good Clinical Practice (as adapted by local health authorities), with applicable local regulations (including European Directive 2001/20/EC and US Code of Federal Regulations Title 21) and with the ethical principles laid down in the Declaration of Helsinki. The study is registered at http://www.clinicaltrials.gov (NCT01351675).

\section{Laboratory Measurements}

Key laboratory assessments were done during screening and at all in-person visits starting at Day 1 (day of the first dose of study drug). These assessments include serum pregnancy tests in women of childbearing potential; clinical chemistries including albumin (determined photometrically), transaminases, blood urea nitrogen, calcium, creatinine (enzymatic), eGFR (4-variable MDRD calculation using enzymatic creatinine determination [22]), total and direct bilirubin, $\gamma$-glutamyl transferase, lactate dehydrogenase, magnesium, phosphorus, uric acid, and $\mathrm{HbA}_{1 c}$; urine for urinalysis and microscopy (only during screening), first morning void for ACR (3 during screening; 2 prior to each in-person study visit); hematology with differential; hepatitis B surface antigen (only performed at screening if lacking evidence of a negative assay in the past year); troponin-T and BNP (Day 1 only). At semi-annual visits only (every 24 weeks starting at Day 1 ), the following will be measured in addition to the laboratory variables described above: lipid panel and plasma and urine samples for biomarkers (if consent given). Blood draws for plasma concentration (pharmacokinetic (PK) samples) of study drug will occur at weeks 4 and 12, and at semi-annual visits thereafter (starting at Week 24).

All blood and urine specimens are shipped at ambient temperature (except for PK and biomarker samples, which are shipped frozen) to central laboratories around the world using an overnight commercial carrier. Validations for laboratory measurements took place at the US site and global correlations occurred for all other locations. Biomarker samples are stored at $-80^{\circ} \mathrm{C}$.

\section{Definition of ESRD and other Endpoints}

For purposes of endpoint adjudication (to be performed by a blinded and independent events adjudication committee (EAC)), ESRD is defined as the need for maintenance dialysis (peritoneal or hemodialysis) for $\geq 12$ weeks or kidney transplantation, or death due to kidney failure. The 12-week duration criterion was incorporated to avoid misclassification of ESRD events requiring acute dialysis resulting from either or both acute kidney injury or acute fluid overload. If ESRD is reached $<12$ weeks before the study termination date, or if a subject dies $<12$ weeks after dialysis initiation, the EAC will adjudicate whether the event represented ESRD or not. An EAC charter documents instructions for adjudication and presents detailed definitions for all renal and CV endpoints.

\section{End of Study}

The end of study is defined as the last post-treatment visit occurring after the SDT date. SDT is determined as the date when approximately 300 patients have experienced a primary endpoint as evaluated by the EAC. The primary efficacy analysis includes all events up to the SDT date. All primary endpoints, including those occurring after the SDT, will be assessed in a sensitivity analysis. While the BEACON Steering Committees remain blinded to the actual results, the trial was terminated prematurely following a recommendation from the independent data monitoring committee. 


\section{Statistical Considerations}

\section{Estimation of Placebo Event Rate}

We built a model that calculated the proportion of patients, based upon baseline eGFR, who would reach dialysis, assuming a mean \pm SD dialysis initiation threshold of $11.1 \pm 4.8 \mathrm{ml} / \mathrm{min} / 1.73 \mathrm{~m}^{2}$ [23] and the eGFR decline rate reported in the placebo group of a previous trial of bardoxolone methyl. Assuming equal distribution of study subject enrollment across a baseline eGFR of 15 to $<30 \mathrm{ml} / \mathrm{min} / 1.73 \mathrm{~m}^{2}, 23 \%$ of patients are expected to initiate maintenance dialysis over 24 months of follow-up. In addition, trials with roughly similar inclusion criteria indicate that on average $21 \%$ of patients are expected to experience ESRD and 5\% CV death over 24 months $[3,4,8,13,24-30]$. Thus, the $5 \% \mathrm{CV}$ death rate and 21 or $23 \%$ ESRD rate from literature sources and deterministic modeling led to a range of 24-month event rates for the composite endpoint (ESRD $+\mathrm{CV}$ death) of $26-28 \%$. Although this estimate reflects the best available data, we recognize some limitations to the literature sources. First, several studies were conducted more than 10 years ago and some improvements may have occurred in standard of care over time. Secondly, there are only limited data about the specific subject population studied in BEACON (i.e. stage $4 \mathrm{CKD}$ with varying levels of albuminuria). Thus, on the basis of the literature described above and output of the deterministic model described below, we predict a slightly lower placebo event rate of $24 \%$ over 24 months for BEACON. We anticipate approximately $75 \%$ of the composite endpoint events will be attributed to ESRD, and $25 \%$ to CV death.

Deterministic Model to Predict Composite Outcome We developed a deterministic model to predict likelihood of dialysis or death in placebo- and bardoxolone methyl-treated patients. The model is based on the premise that rate of dialysis events is driven by the proportion of patients with appreciable eGFR loss, which we defined as $\geq 3 \mathrm{ml} / \mathrm{min} / 1.73 \mathrm{~m}^{2}$ per year from baseline.

Three inputs were used for the model: (1) anticipated distribution of patients with macroalbuminuria at baseline, (2) proportion of patients with appreciable eGFR loss within those subgroups, and (3) their respective rates of eGFR decline. We used likelihood of eGFR decline among patients with macroalbuminuria, microalbuminuria or without measurable albuminuria from a Phase $2 b$ trial of bardoxolone methyl in patients with stage $3 \mathrm{~b} / 4$ CKD and type 2 diabetes mellitus [21]. We included pro- jections for duration of enrollment (14 months), study drug discontinuation (13.5\% per year on study), and loss to follow-up (2.5\% per year). We took into account the possibility that discontinuation from study drug would be more common in bardoxolone methyl-treated patients. To be conservative, we assumed no effect of bardoxolone methyl on CV death.

Model output: Using specified rates of eGFR decline in patients with type 2 diabetes mellitus and stage 4 CKD with and without macroalbuminuria, the model predicted eGFR over time for declining placebo- and bardoxolone methyl-treated patients whose baseline eGFR values are assumed to be uniformly distributed throughout the 15 to $<30 \mathrm{ml} / \mathrm{min} / 1.73 \mathrm{~m}^{2}$ range. Taking into account time on study, the model predicted if and when a dialysis event occurred by noting when eGFR would be expected to fall below the specified dialysis initiation threshold (see above). The model estimated the number of dialysis events occurring each month in the placebo and bardoxolone methyl treatment arm to obtain the estimated drugattributable risk reduction.

Simulations: We previously showed a large discrepancy between time to reach a fixed eGFR value and time to initiation of dialysis [31]. To account for this discrepancy and inherent variability, Monte Carlo simulations were used to stochastically vary eGFR thresholds for initiating dialysis. Several scenarios were modeled including different thresholds for initiating dialysis in placebo and bardoxolone methyl-treated patients, since several previously reported side effects of bardoxolone methyl might be confused with signs of uremia (e.g. muscle cramps, dysgeusia, and weight loss). For each scenario, the frequency distribution from 1,000 trials was used to estimate drug risk reduction for the primary outcome.

Results of the model building and simulations showed an estimated risk reduction for dialysis or $\mathrm{CV}$ death with bardoxolone methyl of $44 \%$ (base case scenario) (table 3; fig. 2). Additional sensitivity analyses of model parameters to simulate multiple worst-case and best-case scenarios showed the minimum expected relative risk reduction with bardoxolone methyl was 33\% (table 3; fig. 2).

\section{Power Calculation}

The power calculation is based on a two-sided logrank statistic tested at a two-sided type I error rate of 0.05 . We will enroll approximately 2,000 patients, anticipating approximately 300 composite primary endpoints during 24 months of follow-up. Under these assumptions, if a log-rank test were used to compare groups, BEACON would have $85 \%$ power to detect a $32 \%$ relative risk reduc- 
Fig. 2. Frequency distribution of simulated overall drug risk reduction for the primary outcome. Each curve represents the predicted drug risk reduction from 1,000 trial simulations of four scenarios described in table 3 .

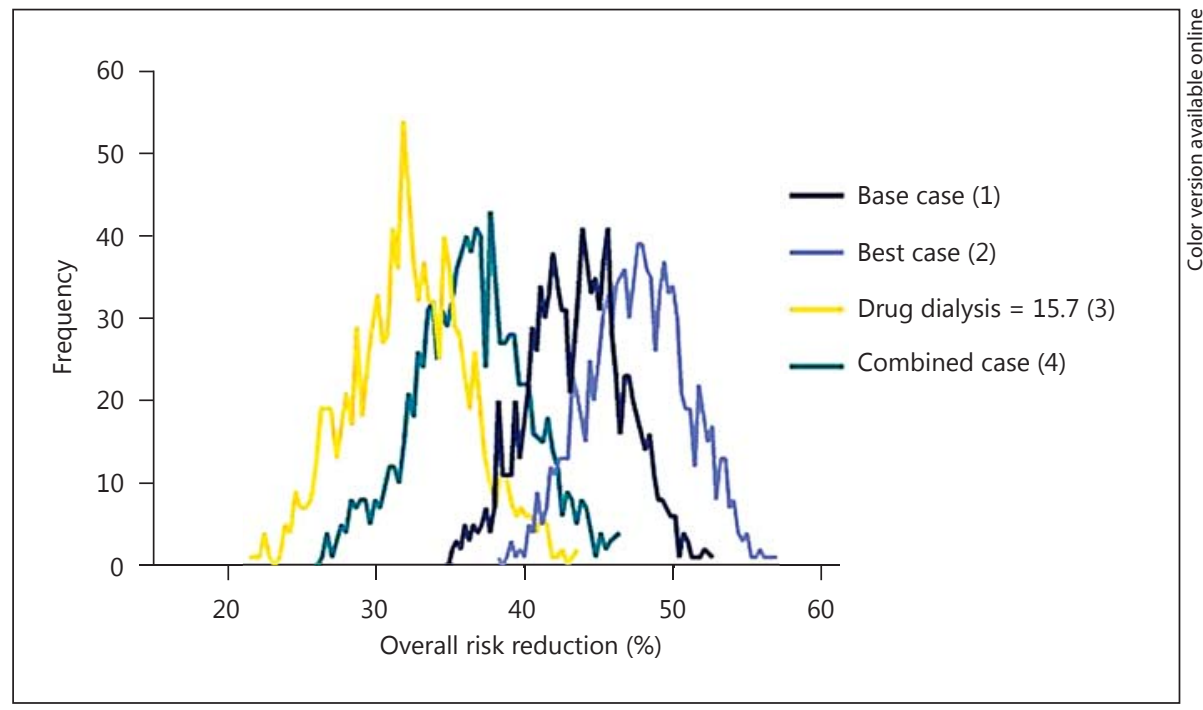

Table 3. Modeling of overall relative risk

\begin{tabular}{|c|c|c|c|}
\hline Scenario & Modified parameter & $\begin{array}{l}\text { Timing of } \\
300 \text { events }\end{array}$ & $\begin{array}{l}\text { Overall RR, \% } \\
(95 \% \text { CI })\end{array}$ \\
\hline Base case & $\begin{array}{l}\text { eGFR decline rate }=\mathrm{M}:-8.0, \mathrm{NM}:-5.5 ; \\
\text { dialysis threshold }(\mathrm{PBO} \text { and drug) }=11.1\end{array}$ & 24 months & $44 \pm 3(38,49)$ \\
\hline $\begin{array}{l}\text { Best case (removing conservative } \\
\text { assumptions) }\end{array}$ & $\begin{array}{l}\text { CV death } \mathrm{RR}=10 \% \text {; } \\
\text { PBO eGFR decline rate: } \mathrm{M}:-10.8, \mathrm{NM}:-8.7 \text {; } \\
\text { drug eGFR decline rate: } \mathrm{M}:-7.4, \mathrm{NM}:-6.5\end{array}$ & +1 week & $48 \pm 3(42,53)$ \\
\hline $\begin{array}{l}\text { Patients on drug start dialysis earlier than } \\
\text { patients on placebo (with base case) }\end{array}$ & dialysis threshold: $\mathrm{PBO}=12.7 ; \mathrm{drug}=15.7$ & -4 weeks & $33 \pm 4(26,39)$ \\
\hline $\begin{array}{l}\text { Patients on drug start dialysis earlier than } \\
\text { patients on placebo (combined with } \\
\text { best case) }\end{array}$ & $\begin{array}{l}\mathrm{CV} \text { death } \mathrm{RR}=10 \% \text {; } \mathrm{PBO} \text { decline rate: } \mathrm{M}:-10.8, \mathrm{NM}:-8.7 \text {; } \\
\text { drug decline rate: } \mathrm{M}:-7.4, \mathrm{NM}:-6.5 ; \\
\text { dialysis threshold: } \mathrm{PBO}=12.7 \text {; drug }=15.7\end{array}$ & -3 weeks & $37 \pm 4(30,43)$ \\
\hline
\end{tabular}

tion (bardoxolone methyl vs. placebo) in incidence of ESRD or CV death. Actual power is likely to be slightly $>85 \%$ because, as described below, the analysis will use a Cox model. If the observed relative risk reduction is roughly $23 \%$, approximately half of the modeled effect, the $\mathrm{p}$ value would be 0.05 .
We pre-specified two pooled, blinded sample size recalculations during BEACON. The first sample size recalculation, conducted after 1,600 patients are randomized, will be used to update sample size model parameters, including enrollment rate, rate of loss to follow-up, discontinuation of study drug rate, eGFR at baseline, and proportion of macroalbuminuric patients. The second sam- 
ple size recalculation, which will assess the event rate for the primary composite outcome event rate and relative proportion of ESRD and CV death events, will be conducted when approximately 200 primary events are adjudicated. The estimated hazard ratio and number of primary events may be updated after one or both sample size recalculations to maintain statistical power.

\section{Efficacy Assessment}

The primary efficacy analysis will be based on a modified intention-to-treat population, defined as all randomized patients who received at least one dose of study drug. The duration of follow-up within BEACON for any given subject depends on the time when the subject enters the study and when the study ends. The primary analysis will employ Cox proportional hazards regression to estimate hazard ratios and 95\% confidence intervals comparing time-to-event outcomes. Because of the importance of UACR and eGFR as predictors of ESRD and CV death, and because of the skewed distribution of UACR, models will be adjusted for log-transformed baseline UACR and baseline eGFR. A log-rank test of unadjusted survival curves will be used as a sensitivity analysis. Kaplan-Meier curves will display the time-to-event.

\section{Safety Assessment}

Safety assessments, including physical examinations, vital sign measurements, centrally read 12-lead electrocardiograms (ECGs), and centrally analyzed clinical laboratory measurements are planned throughout BEACON. Serum creatinine, eGFR, alanine aminotransferase (ALT), and aspartate aminotransferase (AST) values are masked to investigational sites and the sponsor to minimize the potential for unblinding. If ALT or AST values exceed 3 times the upper limit of normal, both the sponsor and site receive unmasked results. If eGFR exceeds $29 \mathrm{ml} / \mathrm{min} / 1.73 \mathrm{~m}^{2}$ for an individual subject, the site is informed to assess the dosage of renally-cleared medications. If required for subject safety, the investigator may request the eGFR, serum creatinine, ALT, or AST value at any time (the sponsor will not receive these values). Concomitant medications are recorded. Frequency, intensity, and relationship to study drug of adverse and serious adverse events, as well as abnormal clinical and laboratory test results, are also evaluated.

\section{Patient-Reported Outcomes}

Health-related quality of life assessments, using the EQ-5D-5L and Kidney Disease Quality of Life (KDQOL) surveys, are collected at baseline and every 24 weeks thereafter.

\section{Discussion}

Residual renal and CV risk remains high in patients with CKD and type 2 diabetes mellitus despite best practice. Recent trials with novel interventional strategies have failed to produce additional renal benefit. Targeting chronic inflammatory and oxidative pathways is a new approach not yet tested in a large-scale outcomes trial in this population. Neither anti-inflammatory drugs nor exogenous addition of antioxidants have proven successful, perhaps because mass endogenous production of reactive oxygen species (ROS) and/or inflammatory mediators was not altered. Bardoxolone methyl may differ as it acts on the endogenous production of ROS and inflammatory pathways simultaneously [32]. By binding KEAP1, it activates Nrf2, the master regulator of the antioxidant response, which induces numerous antioxidant enzymes. Bardoxolone methyl simultaneously downregulates proinflammatory signaling via NF- $\kappa B$ inhibition.

Clinical data on bardoxolone methyl come from two recently published studies in patients with stage $3 \mathrm{~b} / 4$ CKD (eGFR range $\geq 15$ to $<45 \mathrm{ml} / \mathrm{min} / 1.73 \mathrm{~m}^{2}$ ) and type 2 diabetes mellitus: a Phase 2a study and the Bardoxolone Methyl Treatment: Renal Function in CKD/Type 2 Diabetes (BEAM) study $[20,21]$. The BEAM study demonstrated bardoxolone methyl had several effects sustained over at least 1 year, including a mean eGFR increase of $8.1-9.4 \mathrm{ml} / \mathrm{min} / 1.73 \mathrm{~m}^{2}$ in the two higher-dose groups. Whether these effects translate into longer-term benefits is unknown. Complicating the picture is the observed increase in albuminuria. A combination of an early rise in eGFR and increase in albuminuria may not be renoprotective in the longterm, as an acute rise in eGFR has been associated with hyperfiltration, renal damage and subsequent rapid eGFR loss [33], whereas high urinary albumin levels have been associated with increased renal damage [34]. Still, BEAM showed the initial eGFR rise in bardoxolone methyl-treated patients was sustained, without a subsequent fall, during the year-long treatment [21], unlike effects observed with amlodipine in AASK [35]. Pharmacologic mechanisms for the albuminuria increase, such as increases in GFR and tubular reabsorption capacity of albumin, are under active investigation. A recent experimental study showed bardoxolone methyl interferes with megalin expression, which could indicate proximal tubular albumin reabsorption is attenuated [36]. Thus, the albuminuria rise may not be detrimental if albumin is not reabsorbed or bardoxolone methyl interrupts the inflammatory process of excess tubular albumin reabsorption. Results of BEACON will further clarify 
whether the eGFR and albuminuria changes are beneficial or detrimental, or whether bardoxolone methyl added to standard of care will preserve or enhance kidney function to alter ESRD incidence versus standard of care alone.

Theoretically, the eGFR improvement observed in BEAM or other studies may not reflect an improvement in true GFR. However, clinical studies with bardoxolone methyl have shown the decline in serum creatinine is paralleled by a rise in creatinine clearance, with no change in 24-hour urine creatinine excretion, and therefore, no apparent change in creatinine production or breakdown [20]. In addition, eGFR changes inversely correlated with BUN, uric acid, and phosphorus changes [20, 21]. To confirm the effects, two parallel studies are underway testing the effect of bardoxolone methyl on GFR measured with exogenous filtration tracers, one in the US (NCT01500798) and one in Japan.

One dose arm of BEAM showed a small, but statistically significant, rise in blood pressure. Although not observed in other pre-clinical or clinical studies, it could be a safety signal. We are conducting a 24 -hour blood pressure measurement substudy to more definitively assess blood pressure effects of bardoxolone methyl.

Using an ESRD endpoint in a trial with a drug that affects serum creatinine leads to other challenges. First, no recent trial data are available that allow us to estimate accurately ESRD incidence in a stage 4 CKD diabetic population. Second, to estimate the potential effect of bardoxolone methyl on need for dialysis or transplantation, we needed to consider how the drug might be expected to affect eGFR, and to what degree eGFR informs the decision to proceed with dialysis or transplantation. Although central laboratory serum creatinine and eGFR values obtained during BEACON are masked to investigators, site personnel may possibly gain knowledge of these data through other means. Even in the absence of previous trials and drugs that increase eGFR, one can estimate, extrapolate, and calculate the potential slowing of the time to ESRD. However, we needed to take into account the relation between eGFR and ESRD is not straightforward: ESRD is defined as initiation of maintenance dialysis or renal transplant, and decision to start renal replacement therapy is driven not only by eGFR but also other laboratory measures and clinical signs of uremia [37]. Since bardoxolone methyl may also affect parameters other than eGFR (e.g. muscle cramps, taste disturbances) that could mimic uremia, reasons for initiation of dialysis will be documented in each instance, which will help clarify the primary results of the trial.
In summary, administration of bardoxolone methyl has resulted in eGFR increases in several early phase trials. The adverse event profile associated with bardoxolone methyl in prior studies had not demonstrated evidence of any significant harm or resulted in adverse renal effects; however, per-subject exposure has been limited to 1 year. BEACON will test whether this possibly salutary effect on kidney function translates into a delay in progression to ESRD and/or a reduction in CV death or other CV events and will provide additional safety data relevant to bardoxolone methyl and potentially to related drugs.

\section{Steering Committee Members of the BEACON Trial}

Dick de Zeeuw, Glenn M. Chertow (co-chairs), Tadao Akizawa, Paul Audhya, George L. Bakris, Melissa Krauth, Colin J. Meyer, John J. McMurray, Giuseppe Remuzzi, Robert D. Toto, Nosratola D. Vaziri, Christoph Wanner, Hans-Henrik Parving, and Janet Wittes; Hiddo J. Lambers-Heerspink (external advisor).

\section{Termination of the BEACON Trial}

While the BEACON Steering Committee remains blinded to the actual results, the trial was terminated prematurely following a recommendation from the independent data monitoring committee.

\section{Event Adjudication Committee Members of the BEACON Trial}

Rajiv Agarwal (chair), Stefan Anker, Juan Francisco Arenillas, James Januzzi, Alan Jardine, Scott Kasner, Brett Kisslea, Daniel Kolansky, Johannes Mann, and Ravi Thadhani.

\section{Independent Data Monitoring Committee Members of the BEACON Trial}

Peter McCullough (chair), Peter Ivanovich, Markus Ketteler, John Lachin, and Janet McGill.

\section{Contributors to This Communication}

All authors were involved in the design of BEACON, as well as James Stolzenbach (JS). MC, HJLH, JW and Yili Pritchett (YP) provided statistical support. DdZ and GMC wrote the draft of the report, and all authors contributed to its revision. DdZ and GMC take responsibility for the report. Stacey Ruiz (SR) provided editorial assistance, and Ritu Rajan (RR), Angie Goldsberry (AG), Heidi M. Christ-Schmidt (HMCS), and Danielle M. Wrolstad (DMW) provided assistance on the statistical modeling and design. 


\section{Disclosure Statement}

DdZ is a consultant and received honoraria (to employer/institution) from Abbott, Astra-Zeneca, BMS, Novartis, Jansen Pharmaceutica (J\&J), and REATA.

TA is consultant and received grants and honoraria from Kyowa Hakko Kirin, Chugai, Abbott, Bayer and REATA.

RA is a consultant to Merck, Abbott, Celgene, Roche, SigmaTau, and has received research support from Daiichi-Sankyo, Inc.

GLB is a consultant for Takeda, Abbott, CVRx, Johnson \& Johnson, Eli Lilly, Food and Drug Administration, Relapsya and Medtronic. He has received research support (to employer/institution: University of Chicago) from Forest Labs and Takeda.

HJLH is consultant and received honoraria (to employer/institution) from Abbott, Johnson\&Johnson, REATA, and Vitae.

$\mathrm{JJMCM}$ is a consultant and received honoraria (to employer/ institution: Glasgow University) from REATA for his time working as a member of the BEACON Steering Committee.

$\mathrm{H}-\mathrm{HP}$ is consultant and received honoraria from Abbott, Novartis and REATA.

$\mathrm{PEP}$ is an investigator in BEACON and other bardoxolone methyl studies, is a consultant for REATA, and received honoraria from Abbott, Amgen and Vifor Pharma.

GR is consultant for Abbott, REATA and Alexion Pharmaceuticals and received honoraria (to institution and educational activities).
RDT is a consultant for and has received honoraria from Amgen, Boehringer-Ingelheim, Abbott, F. Hoffman-La Roche, and Merck. He has received research support from Novartis and REATA.

NDV has received research funding from REATA.

$\mathrm{CW}$ is consultant and received grants (to employer/institution) and honoraria from Abbott, Amgen, Boehringer-Ingelheim, Genzyme, and REATA.

DGW is a consultant to, and has an equity interest in REATA.

JW is consultant to and has received fees and honoraria (to institution/company: Statistics Collaborative, Inc.) from REATA. Her company, Statistics Collaborative, Inc., has served as the statistical group for the BEACON study and has contracts with many pharmaceutical companies.

GMC serves on the Board of Directors of Satellite Healthcare and the Scientific Advisory Board of DaVita Clinical Research. He has received research support from NIDDK and NHLBI, Amgen and REATA and serves as an advisor to Allocure, Ardelyx, Hemodialysis Plus, Keryx, PuraCath, Theravance and Thrasos Innovations.

PA, MK, CJM, MC, SR, RR and AG are employed by REATA. JS and YP are employed by Abbott Laboratories.

HMCS and DMW are employed by Statistics Collaborative, Inc.

\section{References}

1 US Renal Data System, USRDS 2011 Annual Data Report: Atlas of Chronic Kidney Disease and End-Stage Renal Disease in the United States. Bethesda, National Institutes of Health, National Institute of Diabetes and Digestive and Kidney Diseases, 2011.

$\checkmark 2$ Heerspink HJ, de Zeeuw D: The kidney in type 2 diabetes therapy. Rev Diabet Stud 2011; 8:392-402.

-3 Brenner BM, Cooper ME, de Zeeuw D, Keane WF, Mitch WE, Parving HH, et al: Effects of losartan on renal and cardiovascular outcomes in patients with type 2 diabetes and nephropathy. N Engl J Med 2001;345:861-869.

-4 Lewis EJ, Hunsicker LG, Clarke WR, Berl T, Pohl MA, Lewis JB, et al: Renoprotective effect of the angiotensin-receptor antagonist irbesartan in patients with nephropathy due to type 2 diabetes. N Engl J Med 2001;345:851860.

5 Yusuf S, Sleight P, Pogue J, Bosch J, Davies R, Dagenais G: Effects of an angiotensin-converting-enzyme inhibitor, ramipril, on cardiovascular events in high-risk patients. The Heart Outcomes Prevention Evaluation Study Investigators. N Engl J Med 2000;342: 145-153.

$\checkmark 6$ Gaede P, Lund-Andersen H, Parving HH, Pedersen O: Effect of a multifactorial intervention on mortality in type 2 diabetes. $\mathrm{N}$ Engl J Med 2008;358:580-591.
7 Dahlof B, Devereux RB, Kjeldsen SE, Julius S, Beevers G, De FU, et al: Cardiovascular morbidity and mortality in the Losartan Intervention For Endpoint reduction in hypertension study (LIFE): a randomised trial against atenolol. Lancet 2002;359:995-1003.

-8 Baigent C, Landray MJ, Reith C, Emberson J, Wheeler DC, Tomson C, et al: The effects of lowering LDL cholesterol with simvastatin plus ezetimibe in patients with chronic kidney disease (Study of Heart and Renal Protection): a randomised placebo-controlled trial. Lancet 2011;377:2181-2192.

-9 Lewis EJ, Lewis JB, Greene T, Hunsicker LG, Berl T, Pohl MA, et al: Sulodexide for kidney protection in type 2 diabetes patients with microalbuminuria: a randomized controlled trial. Am J Kidney Dis 2011;58:729-736.

10 Lewis EJ, Greene T, Spitalewiz S, Blumenthal S, Berl T, Hunsicker LG, et al: Pyridorin in type 2 diabetic nephropathy. J Am Soc Nephrol 2012;23:131-136.

11 Mann JF, Schmieder RE, McQueen M, Dyal L, Schumacher H, Pogue J, et al: Renal outcomes with telmisartan, ramipril, or both, in people at high vascular risk (the ONTARGET study): a multicentre, randomised, doubleblind, controlled trial. Lancet 2008;372:547553.
12 Mann JF, Green D, Jamerson K, Ruilope LM, Kuranoff SJ, Littke T, et al: Avosentan for overt diabetic nephropathy. J Am Soc Nephrol 2010;21:527-535.

13 Pfeffer MA, Burdmann EA, Chen CY, Cooper ME, de Zeeuw D, Eckardt KU, et al: A trial of darbepoetin alfa in type 2 diabetes and chronic kidney disease. N Engl J Med 2009;361: 2019-2032.

14 Parving HH, Brenner BM, McMurray JJ, de Zeeuw D, Haffner SM, Solomon SD, et al: Aliskiren Trial in Type 2 Diabetes Using Cardio-Renal Endpoints (ALTITUDE): rationale and study design. Nephrol Dial Transplant 2009;24:1663-1671.

15 Kelly KJ, Dominguez JH: Rapid progression of diabetic nephropathy is linked to inflammation and episodes of acute renal failure. Am J Nephrol 2010;32:469-475.

16 Wu CC, Sytwu HK, Lin YF: Cytokines in diabetic nephropathy. Adv Clin Chem 2012;56: 55-74.

17 Luis-Rodriguez D, Martinez-Castelao A, Gorriz JL, De-Alvaro F, Navarro-Gonzalez JF: Pathophysiological role and therapeutic implications of inflammation in diabetic nephropathy. World J Diabetes 2012;3:7-18.

18 Miyamoto T, Carrero JJ, Stenvinkel P: Inflammation as a risk factor and target for therapy in chronic kidney disease. Curr Opin Nephrol Hypertens 2011;20:662-668. 
19 Anders HJ, Ryu M: Renal microenvironments and macrophage phenotypes determine progression or resolution of renal inflammation and fibrosis. Kidney Int 2011;80:915-925.

-20 Pergola P, Krauth M, Huff W, Ferguson D, Ruiz S, Meyer C, et al: Effect of bardoxolone on kidney function in patients with T2D and stage 3b-4 CKD. Am J Nephrol 2011;33:469476.

21 Pergola PE, Raskin P, Toto RD, Meyer CJ, Huff JW, Grossman EB, et al: Bardoxolone methyl and kidney function in CKD with type 2 diabetes. N Engl J Med 2011;365:327-336.

-22 Stevens LA, Coresh J, Feldman HI, Greene T, Lash JP, Nelson RG, et al: Evaluation of the modification of diet in renal disease study equation in a large diverse population. J Am Soc Nephrol 2007; 18:2749-2757.

23 US Renal Data System, USRDS 2008 Annual Data Report: Atlas of Chronic Kidney Disease and End-Stage Renal Disease in the United States. Bethesda, National Institutes of Health, National Institute of Diabetes and Digestive and Kidney Diseases, 2008.

24 Chiu YL, Chien KL, Lin SL, Chen YM, Tsai TJ, Wu KD: Outcomes of stage 3-5 chronic kidney disease before end-stage renal disease at a single center in Taiwan. Nephron Clin Pract 2008;109:c109-c118.
25 Demoulin N, Beguin C, Labriola L, Jadoul M: Preparing renal replacement therapy in stage 4 CKD patients referred to nephrologists: a difficult balance between futility and insufficiency. A cohort study of 386 patients followed in Brussels. Nephrol Dial Transplant 2011;26:220-226.

26 Drueke TB, Locatelli F, Clyne N, Eckardt KU, Macdougall IC, Tsakiris D, et al: Normalization of hemoglobin level in patients with chronic kidney disease and anemia. N Engl J Med 2006;355:2071-2084.

27 Eddington H, Hoefield R, Sinha S, Chrysochou C, Lane B, Foley RN, et al: Serum phosphate and mortality in patients with chronic kidney disease. Clin J Am Soc Nephrol 2010; 5:2251-2257.

28 Landray MJ, Emberson JR, Blackwell L, Dasgupta T, Zakeri R, Morgan MD, et al: Predic tion of ESRD and death among people with CKD: the Chronic Renal Impairment in Birmingham (CRIB) prospective cohort study. Am J Kidney Dis 2010;56:1082-1094.

29 Nakayama M, Sato T, Sato H, Yamaguchi Y, Obara K, Kurihara I, et al: Different clinical outcomes for cardiovascular events and mortality in chronic kidney disease according to underlying renal disease: the Gonryo Study. Clin Exp Nephrol 2010;14:333-339.

- 30 Obi Y, Kimura T, Nagasawa Y, Yamamoto R, Yasuda K, Sasaki K, et al: Impact of age and overt proteinuria on outcomes of stage 3-5 chronic kidney disease in a referred cohort. Clin J Am Soc Nephrol 2010;5:1558-1565.
31 Weldegiorgis MT, Lambers Heerspink HJ, de Zeeuw D, et al: Is ESRD/renal replacement therapy a precise clinical meaningful renal endpoint in drug trials? (Abstract) J Am Soc Nephrol 2012;23 (404A).

32 Sporn MB, Liby KT, Yore MM, Fu L, Lopchuk JM, Gribble GW: New synthetic triterpenoids: potent agents for prevention and treatment of tissue injury caused by inflammatory and oxidative stress. J Nat Prod 2011;74:537545 .

33 Heerspink HJ, Holtkamp FA, de Zeeuw D, Ravid M: Monitoring kidney function and albuminuria in patients with diabetes. Diabetes Care 2011;34(suppl 2):S325-S329.

34 Abbate M, Zoja C, Remuzzi G: How does proteinuria cause progressive renal damage? J Am Soc Nephrol 2006;17:2974-2984.

- 35 Agodoa LY, Appel L, Bakris GL, Beck G, Bourgoignie J, Briggs JP, et al: Effect of ramipril vs. amlodipine on renal outcomes in hypertensive nephrosclerosis: a randomized controlled trial. JAMA 2001;285:2719-2728.

36 Reisman SA, Chertow GM, Hebbar S, Vaziri ND, Ward KW, Meyer CJ: Bardoxolone methyl decreases megalin and activates Nrf2 in the monkey kidney. J Am Soc Nephrol 2012;23:1663-1673.

37 K/DOQI Clinical Practice Guidelines and Clinical Practice Recommendations for 2006 Updates: Hemodialysis Adequacy, Peritoneal Dialysis Adequacy and Vascular Access. Am J Kidney Dis 2006;48(suppl 1):S1-S322. 